\title{
Is it Possible to Successfully Treat Locally Advanced Colon Cancer Using Pre-Operative Chemoradiotherapy?
}

\author{
Ji Hun Choi', Jae Hyun Kim', Won Moon', Seung Hun Lee², Sung Uhn Baek², Byung Kwon Ahn², Jung Gu Park and Seun Ja Park' \\ Department of ${ }^{1}$ Internal Medicine, ${ }^{2}$ Colorectal Surgery, ${ }^{3}$ Radiology, Kosin University College of Medicine, Busan, Korea
}

Pre-operative chemoradiotherapy (CRT) is a preferable treatment option for patients with locally advanced rectal cancer. However, few data are available regarding pre-operative CRT for locally advanced colon cancer. Here, we describe two cases of successful treatment with pre-operative CRT and establish evidence supporting this treatment option in patients with locally advanced colon cancer. In the first case, a 65-year-old woman was diagnosed with ascending colon cancer with duodenal invasion. In the second case, a 63-year-old man was diagnosed with a colonic-duodenal fistula due to transverse colon cancer invasion. These case reports will help to establish a treatment consensus for pre-operative CRT in patients with locally advanced colon cancer. Clin Endosc 2019;52:191-195

Key Words: Colon cancer duodenal invasion; Locally advanced colon cancer; Pre-operative chemoradiotherapy

\section{INTRODUCTION}

Colorectal cancer (CRC) is among the most common cancers and the third leading cause of cancer-related death worldwide. ${ }^{1}$ Locally advanced CRC, which infiltrates adjacent organs or structures, is present at diagnosis in approximately $5 \%-16.7 \%$ of cases. $^{2,3}$ The treatment of locally advanced CRC depends on the tumor location. The standard treatments are surgical resection for colon cancer and neoadjuvant chemoradiotherapy (CRT) for rectal cancer. ${ }^{4}$ Pre-operative CRT is preferred for patients with locally advanced rectal cancer because it is associated with reduced toxicity and improved rates of local control and sphincter preservation. ${ }^{5}$ However, few data are available regarding pre-operative CRT for locally advanced colon cancer. The current National Comprehensive

Received: May 29, 2018 Revised: August 3, 2018

Accepted: August 8, 2018

Correspondence: Seun Ja Park

Department of Internal Medicine, Kosin University College of Medicine, 262 Gamcheon-ro, Seo-gu, Busan 49267, Korea

Tel: +82-51-990-6103, Fax: +82-51-990-5055, E-mail: parksj6406@hanmail.net ORCID: https://orcid.org/0000-0003-3217-5115

cc This is an Open Access article distributed under the terms of the Creative Commons Attribution Non-Commercial License (http://creativecommons.org/ licenses/by-nc/3.0) which permits unrestricted non-commercial use, distribution, and reproduction in any medium, provided the original work is properly cited.
Cancer Network (NCCN) guidelines suggest pre-operative CRT as an option for facilitating the resectability of initially unresectable non-metastatic T4 colon cancer. ${ }^{6}$ Herein, we describe two cases of successful treatment with pre-operative CRT and establish evidence for pre-operative CRT in patients with locally advanced colon cancer.

\section{CASE REPORTS}

\section{Case 1}

A 65-year-old Korean woman presented to the emergency room at Kosin University Gospel Hospital for the management of melena and abdominal pain that had persisted for 2 weeks. Two months before presenting at our hospital, she had experienced fatigue and dizziness. At presentation, she was dizzy and had abdominal pain. She had no fever, chills, body weight loss, nausea, vomiting, or changes in bowel habits. Her vital signs were normal. She complained of some pain in the right upper quadrant but had no tenderness or rebound tenderness during a physical examination. Her hemoglobin level had decreased to $5.6 \mathrm{~g} / \mathrm{dL}$. A computed tomography (CT) scan showed fat stranding around the ascending colon with irregular wall thickening and a low-density, $1.5-\mathrm{cm}$ lesion at the duodeno-pancreatic groove (Fig. 1A). Metastatic lymph- 
adenopathy was present in the para-aortic area and hepatoduodenal ligament. A colonoscopic examination revealed an ulcero-infiltrative 4-cm tumor in the hepatic flexure (Fig. 1B). An upper endoscopic examination revealed a large exophytic mass with obstruction in the second duodenal portion (Fig. 1C). Biopsies were obtained from both the colon and duodenum. Histologically, both tumors comprised of adenocarcinoma (K-Ras wild-type, epidermal growth factor receptor-positive); additionally, immunohistochemistry revealed that the duodenal tumor was cytokeratin 7-positive and cytokeratin 20-negative. Positron emission tomography/CT showed localized hypermetabolic wall thickening in the ascending colon and a hypermetabolic lymph node in the duodeno-pancreatic groove, which was considered an invasion of the duodenum. A few hypermetabolic lymph nodes were also present around the right colic vein and abdominal para-aortic area. Based on these findings, a diagnosis of ascending colon cancer with di- rect invasion of the duodenum was made. A multidisciplinary team, including a gastroenterologist, surgeon, radiologist, oncologist, and radiation oncologist, decided to treat this patient with pre-operative CRT and subsequent surgery. The chemotherapy regimen consisted of cetuximab with FOLFIRI, and radiation therapy involved a total dose of 4,500 cGy divided into 25 fractions ( 5 times per week). After the completion of radiation therapy and four cycles of chemotherapy, the patient was reevaluated for tumor resectability. CT imaging indicated a decrease in the irregularly enhanced wall thickening at the ascending colon. The right colic and retropancreatic adenopathy had also decreased in size (Fig. 2A). A colonoscopic and upper endoscopic examination revealed significant reductions in tumors, compared to previous studies (Fig. 2B, C). Four additional cycles of chemotherapy were administered. These additional four cycles led to a total of eight completed chemotherapy cycles, after which another colonoscopic and
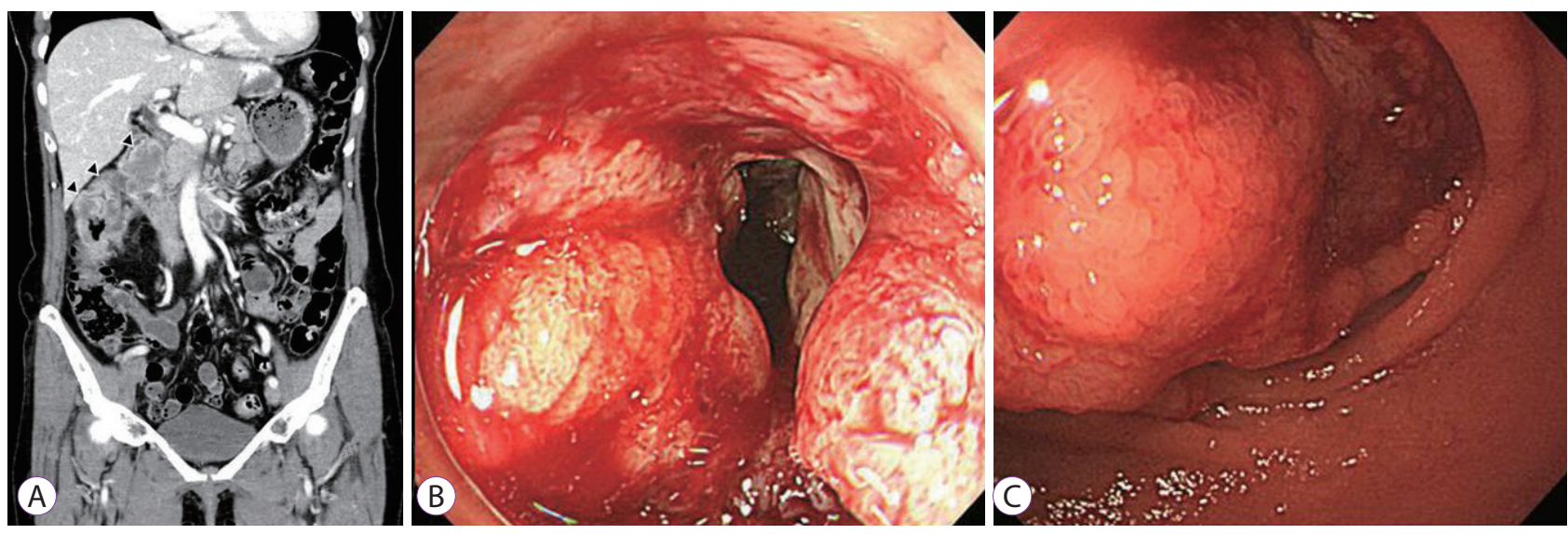

Fig. 1. (A) Abdominal computed tomography revealed fat stranding around the ascending colon with irregular wall thickening and a 1.5-cm, low-density lesion on the duodeno-pancreatic groove (black arrowhead). (B) Colonoscopic examination showed an ulcero-infiltrative mass with luminal narrowing. (C) Upper endoscopic examination showed a large exophytic mass with obstruction in the second portion of the duodenum.
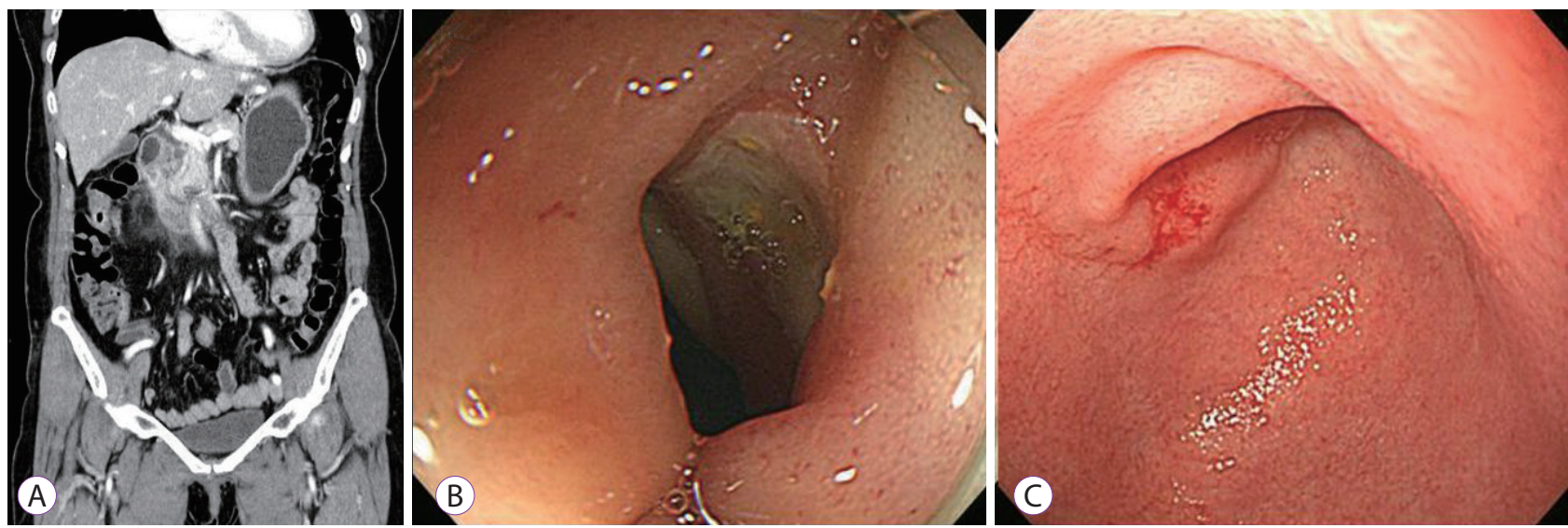

Fig. 2. (A) Abdominal computed tomography revealed reduced irregularly enhancing wall thickening at the ascending colon. (B, C) Colonoscopic and upper endoscopic examination revealed significantly reduced tumors compared to previous studies. 
upper endoscopic examination was performed. Endoscopic biopsy revealed no cancer cells. A laparoscopic extended right hemicolectomy with D3 lymph node dissection was then performed. Fortunately, the duodenum and pancreas were preserved. Histology revealed that the tumor had invaded the pericolic adipose tissue (ypT3), and five out of 15 nodes were found to be invasion positive (ypN2a). All five invaded nodes were pericolic lymph nodes. The surgical resection margins were clear. An additional eight cycles of chemotherapy were performed postoperatively. The patient has remained in good health for 26 months after diagnosis and has not developed a recurrence during the 13 months after ending chemotherapy.

\section{Case 2}

A 63-year-old Korean man visited the clinic at Kosin University Gospel Hospital for the management of a palpable abdominal mass. Upon presentation at the clinic, he was oth- erwise healthy. His carcinoembryonic antigen level was 4.65 $\mathrm{ng} / \mathrm{mL}$ (reference value, $<5.0$ ), and the results of a complete blood count, plasma electrolyte analysis, coagulation analysis, kidney and liver function tests, and urinalysis were normal. A physical examination revealed no other significant findings. Abdominal CT showed a large irregular mass at the transverse colon, which had extended to and invaded the duodenum to form a fistula (Fig. 3A). A colonoscopic examination revealed poorly circumscribed infiltrating masses with ulceration in the transverse colon (Fig. 3B). The mass occupied nearly the entire lumen, which the colonoscope could not access. An upper endoscopic examination revealed a 2 -cm fistula tract covered with yellowish exudate (Fig. 3C). A multidisciplinary team decided to treat this patient with pre-operative CRT and subsequent surgery. The chemotherapy regimen was FOLF$\mathrm{OX} 4$, and radiation treatment was administered at a total dose of 3,000 cGy divided into 10 fractions (5 times per week). Af-
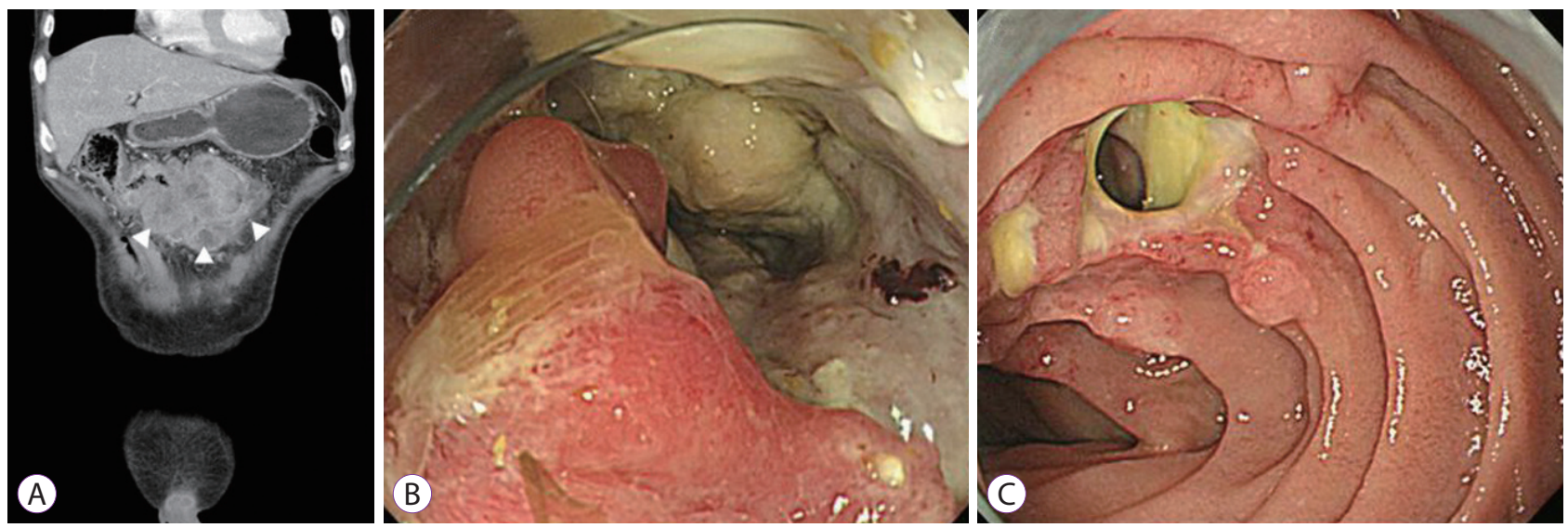

Fig. 3. (A) Computed tomography image showing a large irregular mass at the transverse colon extending to and invading the duodenum. (B) Colonoscopic images showed poorly circumscribed infiltrating masses with ulceration in the transverse colon. (C) Upper endoscopic examination showed a 2-cm fistula tract covered with yellowish exudates.
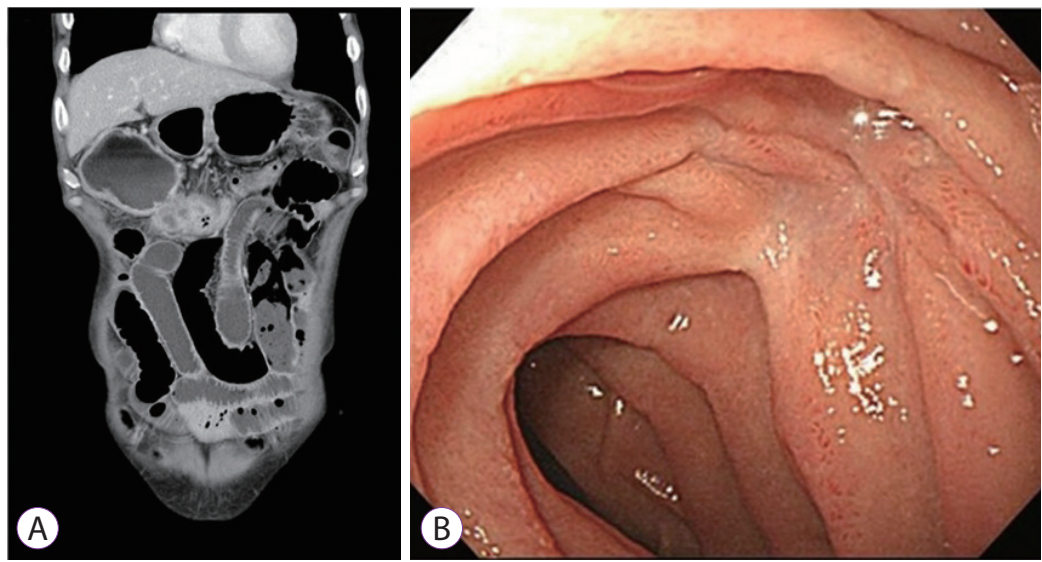

Fig. 4. Abdominal computed tomography revealed decreased size of the enhancing lobulated mass in the transverse colon and alleviated invasion of the adjacent anterior abdominal wall and duodenum (A). Upper endoscopic examination showed that the fistula opening was closed and replaced with a white scar (B). 
ter the completion of chemotherapy and radiation treatment, an abdominal CT and upper endoscopic examination were performed to assess resectability. Abdominal CT revealed that the enhancing lobulated mass in the transverse colon had decreased in size and that the invasion of the adjacent anterior abdominal wall and duodenum had also improved (Fig. 4A). The upper endoscopic examination showed that a whitish scar had replaced the fistula opening, which was now closed (Fig. 4B). The patient suddenly developed abdominal pain while waiting for the scheduled operation, and chest X-ray revealed free air. The patient then underwent an emergency operation; our surgical team, which was aware of his disease status, confirmed that the perforation had occurred in the cancer lesion and performed a transverse colon segmental resection. Histology revealed that the tumor had invaded the muscularis propria (ypT2), with no evidence of lymphovascular invasion (ypN0). After surgery, the patient received an additional 10 cycles of FOLFOX4 chemotherapy. The patient has remained in good health with no recurrence for 54 months after completing treatment.

\section{DISCUSSION}

Preoperative CRT is the gold-standard treatment for locally advanced rectal cancer because it can lead to tumor down-staging and reduce the incidence of local recurrence. ${ }^{4,7}$ Furthermore, a recent NCCN Colorectal Cancer Database study indicated that the number of patients who completed postoperative chemotherapy was significantly lower than the number of those completing pre-operative CRT. ${ }^{8}$ Recently, the field of pre-operative CRT has been extended beyond established therapies, with topics including the identification of patients who have achieved a pathologic complete remission (CR) after treatment and which chemotherapy regimens are associated with a high frequency of pathologic CR. ${ }^{9,10}$ However, radiotherapy has two major limitations when applied to colon cancers. First, colon cancer is a difficult target to define because the colon is a movable organ. Second, the colon is surrounded by dose-limiting structures, including the small bowel, kidney, and liver. ${ }^{11}$ Therefore, even if radiation therapy is selected, the radiation dose is minimized to reduce the risk of complications. In our two cases, the optimal radiation dose was reduced by $11 \%$ and $40 \%$, respectively, compared to the standard radiation dose for rectal cancer. In the second case, although we reduced the therapeutic dose, colonic perforation occurred 20 days after the completion of radiotherapy. This may be attributable to penetration of the tumor through the colon wall and the formation of fistulae into adjacent organs, or to weakening and regression after CRT. Fortunately, the patient recovered without requiring a duodenectomy and remains alive without recurrence.

Patients with locally advanced CRC have good long-term outcomes with complete resection. Previous studies have reported 5-year survival rates of $49 \%-54 \%$ and median survival durations of 38-42 months for patients with locally advanced CRC in which the tumor was completely resected. ${ }^{12-14}$ The long-term outcomes of our patients were similar to those of previous reports.

Radiation therapy plays a well-established role in the complete resection of rectal cancer. In these case reports, we present another possibility, namely that radiation therapy can be administered to patients with locally advanced colon cancer before surgical resection. These case reports will help to establish a treatment consensus for pre-operative CRT in patients with locally advanced colon cancer.

\section{Conflicts of Interest}

The authors have no financial conflicts of interest.

\section{REFERENCES}

1. Bevan R, Rutter MD. Colorectal cancer screening-who, how, and when? Clin Endosc 2018;51:37-49.

2. Govindarajan A, Fraser N, Cranford V, et al. Predictors of multivisceral resection in patients with locally advanced colorectal cancer. Ann Surg Oncol 2008;15:1923-1930.

3. Staniunas RJ, Schoetz DJ Jr. Extended resection for carcinoma of colon and rectum. Surg Clin North Am 1993;73:117-129.

4. National Comprehensive Cancer Network. NCCN clinical practice guidelines in oncology: rectal cancer version 3. 2017 [Internet]. Fort Washington (PA): National Comprehensive Cancer Network; c2017 [cited 2017 Mar 13]. Available from: https://www.nccn.org/professionals/ physician_gls/pdf/rectal.pdf.

5. Sauer R, Becker H, Hohenberger W, et al. Preoperative versus postoperative chemoradiotherapy for rectal cancer. N Engl J Med 2004;351:17311740.

6. National Comprehensive Cancer Network. NCCN clinical practice guidelines in oncology: colon cancer version 2. 2017 [Internet]. Fort Washington (PA): National Comprehensive Cancer Network; c2017 [cited 2017 Mar 13]. Available from: https://www.nccn.org/professionals/ physician_gls/pdf/colon.pdf.

7. Sauer R, Liersch T, Merkel S, et al. Preoperative versus postoperative chemoradiotherapy for locally advanced rectal cancer: results of the German CAO/ARO/AIO-94 randomized phase III trial after a median follow-up of 11 years. J Clin Oncol 2012;30:1926-1933.

8. Khrizman P, Niland JC, ter Veer A, et al. Postoperative adjuvant chemotherapy use in patients with stage II/III rectal cancer treated with neoadjuvant therapy: a national comprehensive cancer network analysis. J Clin Oncol 2013;31:30-38

9. Franke AJ, Parekh H, Starr JS, Tan SA, Iqbal A, George TJ Jr. Total neoadjuvant therapy: a shifting paradigm in locally advanced rectal cancer management. Clin Colorectal Cancer 2018;17:1-12.

10. Kalyan A, Rozelle S, Benson A 3rd. Neoadjuvant treatment of rectal cancer: where are we now? Gastroenterol Rep (Oxf) 2016;4:206-209.

11. Agranovich A, Berthelet E. Radiotherapy for colorectal cancer. B C Med J 2000;42:139-141. 
12. Curley SA, Carlson GW, Shumate CR, Wishnow KI, Ames FC. Extended resection for locally advanced colorectal carcinoma. Am J Surg 1992;163:553-559.

13. Curley SA, Evans DB, Ames FC. Resection for cure of carcinoma of the colon directly invading the duodenum or pancreatic head. J Am Coll
Surg 1994;179:587-592.

14. Taylor WE, Donohue JH, Gunderson LL, et al. The Mayo Clinic experience with multimodality treatment of locally advanced or recurrent colon cancer. Ann Surg Oncol 2002;9:177-185. 